\title{
Acute Stroke of the Insular Cortex Leading to Heart Failure
}

\author{
Isaac Akkad ${ }^{\mathrm{a}, \mathrm{d}}$, Suhali Kundu ${ }^{\mathrm{b}}$, Avraham Miller ${ }^{\mathrm{a}}$, Jyothi Ramachandran ${ }^{\mathrm{b}}$, \\ Vijay Shetty ${ }^{\mathrm{c}}$
}

\begin{abstract}
In humans, the limbic and paralimbic systems, including the insular cortex, are the central autonomic networks responsible for maintaining cardiovascular autonomic control. The right insular cortex plays a predominant role in establishing sympathetic tone while the left insular cortex in establishing parasympathetic tone of the heart. Disturbances in the autonomic system due to cerebrovascular diseases, such as an ischemic stroke in the territory of the middle cerebral artery, could lead to impairment in the cardiovascular balance, consequently increasing the incidence of tachyarrhythmia. Abnormalities of cardiovascular autonomic control may retain prognostic relevance, as they have been associated with adverse clinical outcomes after stroke. Acute ischemic stroke has been shown to increase the rate of cardiac-related mortality as a pathophysiological consequence of an acute stress response in patients with no cardiovascular comorbidities due to the dysfunction of the brain-heart axis. This case report highlights an instance when this axis was disturbed leading to non-ischemic cardiomyopathy.
\end{abstract}

Keywords: Acute stroke; Heart failure; Insular cortex; Non-ischemic cardiomyopathy; Parasympathetic system; Sympathetic system; Right middle cerebral artery

\section{Introduction}

In both animal and clinical studies, it has been shown that disease in the cerebrovascular system can alter cardiovascular function. Stroke has been proven to produce changes in autonomic function, increase the incidence of cardiac arrhythmias

\footnotetext{
Manuscript accepted for publication January 29, 2016

aDepartment of Medicine, Maimonides Medical Center, Brooklyn, NY 11219, USA

bSemmelweis University, Faculty Medicine, Budapest, Ulloi ut 26, Hungary 'Department of Cardiology, Maimonides Medical Center, Brooklyn, NY 11219, USA

${ }^{\mathrm{d} C}$ Corresponding Author: Isaac Akkad, Department of Medicine, Maimonides Medical Center, Brooklyn, NY 11219, USA.

Email: iakkad@maimonidesmed.org
}

doi: http://dx.doi.org/10.14740/jmc2426w and increase the serum levels of catecholamines $[1,2]$. Most deaths occurring after an ischemic stroke can be directly attributed to the neurological injury, but $2-6 \%$ of all stroke patients will die from cardiac causes [3].

In clinical studies, the most important control sites of autonomic function are the insular cortex, amygdala and lateral hypothalamus. Further analysis has shown that strokes in the right hemisphere produce more significant sympathetic effect than left-sided strokes. Localization and mapping of the right hemisphere has actually shown the right insular cortex in particular to be very significant in producing some of the more deadly cardiac arrhythmias after a cerebrovascular accident [4, 5]. This case report will highlight a similar case in which the arrhythmia developed after a stroke and led to further deconditioning and heart failure.

\section{Case Report}

A 58-year-old female presented to the emergency room (ER) with a 4-h history of left-sided weakness and dysarthria. Her past medical history was significant for hypertension, hyperlipidemia, type 2 diabetes and breast cancer for which she underwent bilateral mastectomy and chemotherapy. In addition to the weakness, she was complaining of a day of chest pain and shortness of breath but was denying any other accompanying symptoms. Upon presentation, her vitals were significant only for mild hypotension. A stroke code was immediately called and she had lab work done and was sent for a head CT. Labs including cardiac enzymes were unremarkable. Initially, the head CT was within normal limits, but a repeat CT showed a new hypodensity in the right posterior insular cortex (Fig. 1, 2). MRI was unable to be performed in this patient due to implants. EKG on admission showed a prolonged QTc, poor R wave progression, Q waves (Fig. 3). Echocardiogram (TTE) showed a left ventricular ejection fraction of $40 \%$ with global cardiomyopathy. She was admitted and treated medically, and her weakness resolved. During her stay, she had multiple runs of non-sustained ventricular tachycardia and upon discharge, she was sent home with a beta-blocker, aspirin, high-dose statin and a life vest.

Three weeks later, she presented back to the ER with 5 days of shortness of breath and chest pain after she had a recent viral illness. Upon arrival, her vitals were significant for tachycardia into the $120 \mathrm{~s}$. Her labs were significant for an elevated 


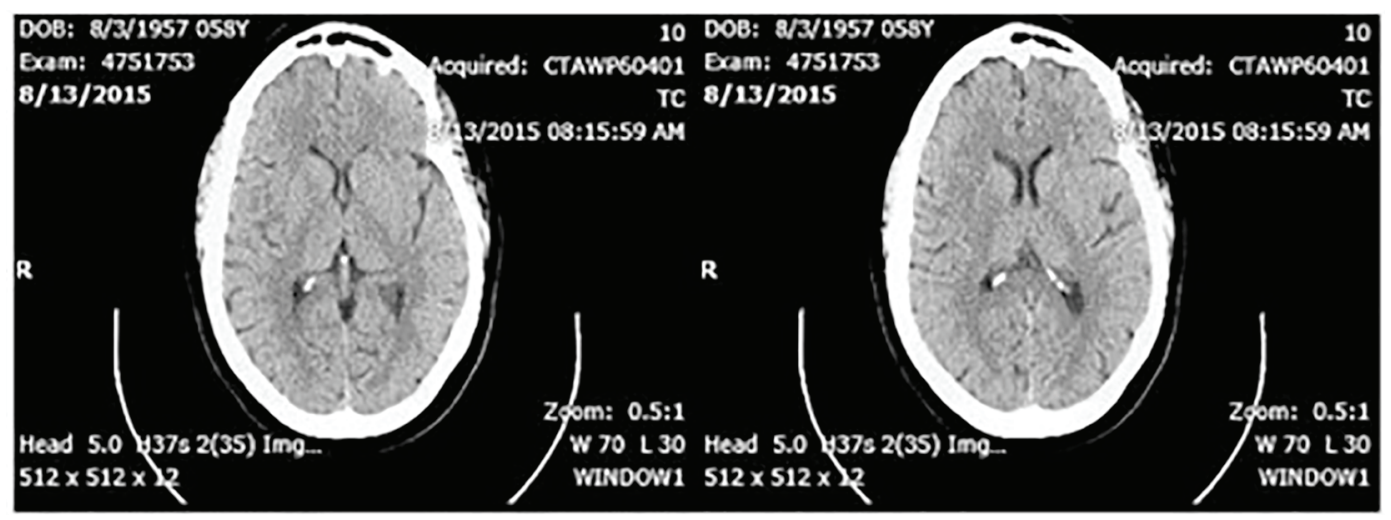

Figure 1. Initial CT scan with no IV contrast.

B-type natriuretic peptide. During her stay, she had multiple runs of premature ventricular complexes and paroxysmal atrial fibrillation. The patient was sent for a coronary angiogram that showed normal coronary vessels. She was treated with amiodarone and digoxin and then evaluated by electrophysiology regarding the placement of an internal defibrillator. Due to her ejection fraction being above $35 \%$, the team felt she did not qualify for the defibrillator and was sent home with her betablocker, amiodarone and apixaban once she was diuresed and asymptomatic.

\section{Discussion}

In humans, stroke in both hemispheres has been shown to produce changes in autonomic function leading to arrhythmias and even sudden cardiac death. In a study by Lane et al, it was determined that right hemisphere infarction is associated with a greater number of supraventricular tachycardia (SVT). They speculated that this was due to a decrease in parasympathetic activity in the right-sided infarcts leading to a reciprocal rise in sympathetic tone [6]. Of all the areas involved in autonomic function, studies have shown the insular cortex to play a major role. This area within the middle cerebral artery (MCA) territory is of particular importance as it has been shown to play a role both in the sympathetic and parasympathetic cardiovascular regulation. Usually the insula is involved in MCA occlusion, which is a major cause of ischemic stroke [5].

It has been shown that focal cerebral ischemia in animals tends to lead to fluctuation in heart rate and blood pressure only if the insular cortex is involved. In fact, stimulation of different areas within the insular cortex will invoke different cardiac autonomic responses. Microstimulation of a rat left insula resulted in QT interval prolongation, ST depression, bradycardia and complete heart block. On examination, the plasma noradrenaline levels were elevated without a change in adrenaline, indicating a neural rather than an adrenal origin [7].

Lesions in the right posterior insula have been shown to increase blood pressure and heart rate without altering baroreceptor sensitivity [8]. Spectral analysis of the heart rate can often be used as a surrogate for measuring sympathovagal balance. Stimulation of the area of the right posterior insular area increases sympathetic tone in the absence of heart rate, blood pressure or respiration changes [9]. However, in a study by $\mathrm{Li}$ et al, it was determined that SVTs were more frequently encountered after right insular infarction compared with strokes in other locations. Additionally, higher serum noradrenaline levels, longer QTc prolongation and more cardiac arrhythmias were observed after a right hemisphere infarction [10]. It is proposed that the sympathetic overactivity and the catecholamine surge following a stroke in the right insular cortex increases myocardial oxygen demand, limiting coronary perfusion. This results in a calcium overload in the ventricular myocytes. This cascade of events is considered pro-arrhythmogenic and can trigger ventricular tachyarrhythmias. Furthermore, in the absence of coronary artery disease, this sympathetic hyperactivity [11] together with reduced cardiac parasympathetic innervation and baroreceptor function prolongs the repolarization

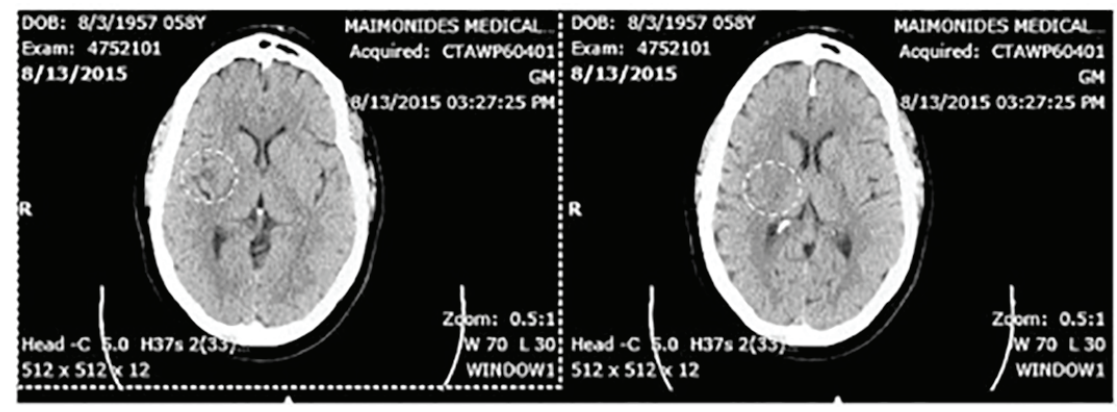

Figure 2. Right insular stroke on CT scan with no IV contrast $6 \mathrm{~h}$ post-presentation (encircled in white). 


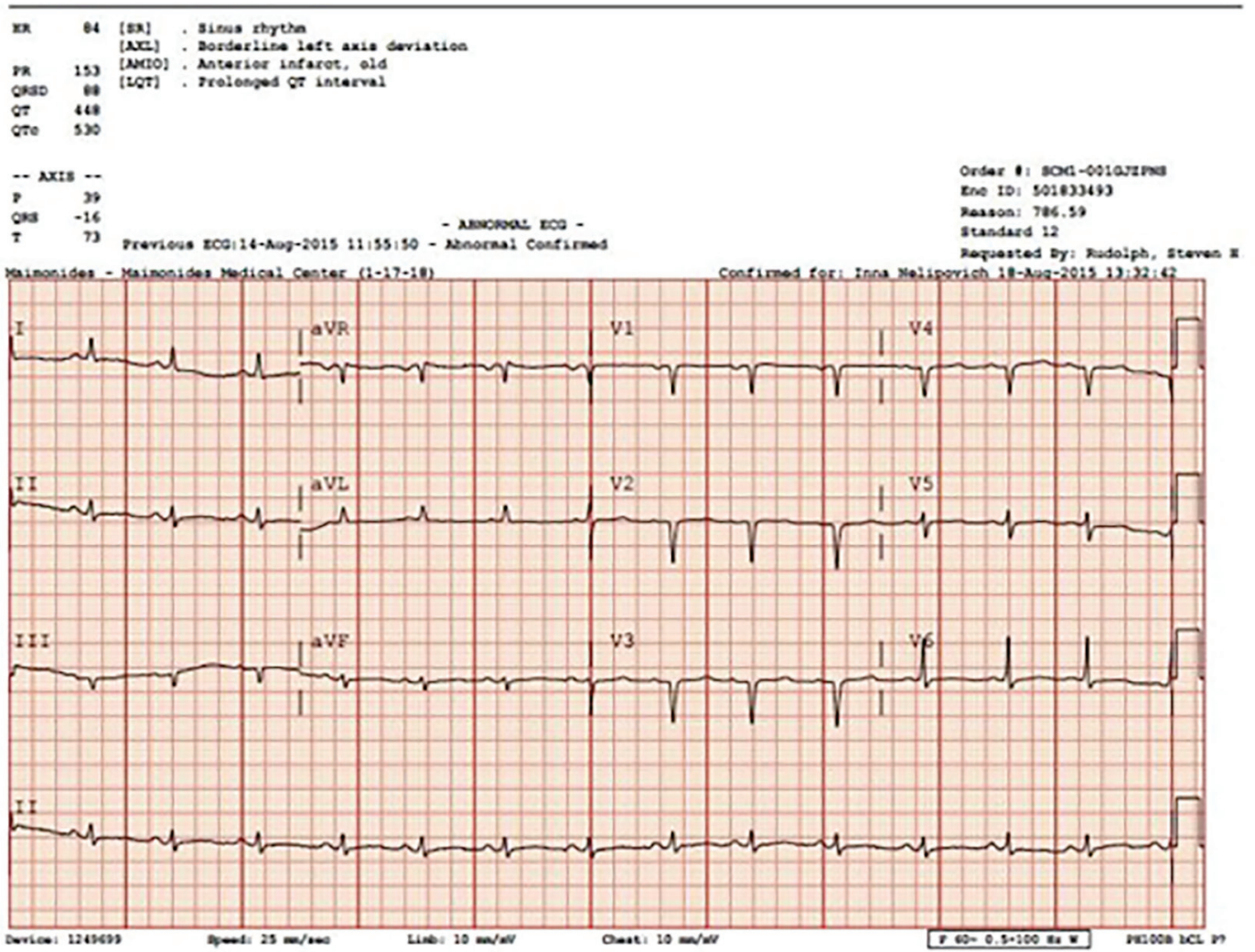

Figure 3. Post-insular stroke ECG showing normal sinus rhythm with prolonged QTC.

duration and accounts for ventricular repolarization changes and increased susceptibility to arrhythmias [12]. Continuous activation of $\beta 1$ adrenergic receptors leads to opening of the calcium channels which induces the stimulation of the actinmyosin complex and causes cardiac muscle contraction with an inability to relax, consequently leading to metabolic imbalance and cell death. When this area of myocyte cell death involves the cardiac conducting system, it frequently presents as QTc prolongation and tachyarrhythmias [13].

The association between tachycardia and cardiomyopathy has been known for some time. Virtually every form of SVT including ectopic atrial tachycardia, junctional tachycardia and atrial fibrillation has been associated with reversible cardiomyopathy. Very frequent ventricular ectopic beats like premature ventricular contractions have also been associated with cardiomyopathy. Chronic tachycardia produces significant cardiac structural changes, including left ventricular dilatation and cellular morphological changes [14]. Our patient presented with the described variability in autonomic function. Following his stroke, the patient was found to have heart rate lability. After a period of time, this continuous onslaught of tachycardia and ventricular ectopy lead to left ventricular dilatation and a reduction in systolic function.

\section{Conclusion}

Cardiac death is the second most common cause of death fol- lowing an acute ischemic cerebrovascular event. This is especially true when there is an ischemic stroke involving the right MCA. Lesions to the right insular cortex lead to a surge of catecholamines leading to heart rate variability and a multitude of tachyarrhythmias. Untreated, the tachycardia will lead to cardiac structural changes and left ventricular dilatation causing a non-ischemic cardiomyopathy. This case highlights the importance of monitoring for and treating tachyarrhythmias in stroke patients especially in the territory of the right MCA. When deemed necessary, these patients would benefit from strict rate control, thus preventing tachycardia-induced cardiomyopathy.

\section{Conflicts of Interest}

The authors declare that there is no conflict of interest regarding the writing of this manuscript.

\section{References}

1. Talman WT. Cardiovascular regulation and lesions of the central nervous system. Ann Neurol. 1985;18(1):1-13.

2. Oppenheimer SM, Cechetto DF, Hachinski VC. Cerebrogenic cardiac arrhythmias. Cerebral electrocardiographic influences and their role in sudden death. Arch Neurol. 1990;47(5):513-519.

3. Adams RJ, Chimowitz MI, Alpert JS, Awad IA, Cerque- 
ria $\mathrm{MD}$, Fayad $\mathrm{P}$, Taubert KA. Coronary risk evaluation in patients with transient ischemic attack and ischemic stroke: a scientific statement for healthcare professionals from the Stroke Council and the Council on Clinical Cardiology of the American Heart Association/American Stroke Association. Stroke. 2003;34(9):2310-2322.

4. Oppenheimer S, Norris JW. Cardiac manifestations of acute neurological lesions. In: MJ Aminoff MJ, ed. Neurology and General Medicine: The Neurological Aspects of Medical Disorder. 2nd ed. New York, NY: Churchill -Livingstone; 1995: 183-200.

5. Korpelainen JT, Sotaniemi KA, Myllyla VV. Asymmetric sweating in stroke: a prospective quantitative study of patients with hemispheral brain infarction. Neurology. 1993;43(6):1211-1214

6. Lane RD, Wallace JD, Petrosky PP, Schwartz GE, Gradman AH. Supraventricular tachycardia in patients with right hemisphere strokes. Stroke. 1992;23(3):362-366

7. Oppenheimer SM, Wilson JX, Guiraudon C, Cechetto DF. Insular cortex stimulation produces lethal cardiac arrhythmias: a mechanism of sudden death? Brain Res. 1991;550(1):115-121.
8. Zhang ZH, Rashba S, Oppenheimer SM. Insular cortex lesions alter baroreceptor sensitivity in the urethane-anesthetized rat. Brain Res. 1998;813(1):73-81.

9. Robinson TG, Dawson SL, Eames PJ, Panerai RB, Potter JF. Cardiac baroreceptor sensitivity predicts longterm outcome after acute ischemic stroke. Stroke. 2003;34(3):705-712.

10. Li C, Dong W. [Abnormal dynamic electrocardiogram in patients with acute cerebral infarction]. Zhonghua Nei Ke Za Zhi. 1999;38(4):239-241.

11. Goldstein DS. The electrocardiogram in stroke: relationship to pathophysiological type and comparison with prior tracings. Stroke. 1979;10(3):253-259.

12. Chalela JA, Ezzeddine MA, Davis L, Warach S. Myocardial injury in acute stroke: a troponin I study. Neurocrit Care. 2004;1(3):343-346.

13. Chua HC, Sen S, Cosgriff RF, Gerstenblith G, Beauchamp NJ, Jr., Oppenheimer SM. Neurogenic ST depression in stroke. Clin Neurol Neurosurg. 1999;101(1):44-48.

14. Shachnow N, Spellman S, Rubin I. Persistent supraventricular tachycardia; case report with review of literature. Circulation. 1954;10(2):232-236. 